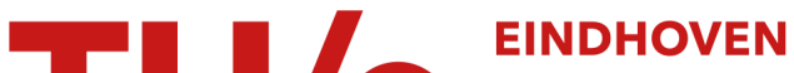 \\ UNIVERSITY OF \\ TECHNOLOGY
}

\section{Highlighting the lambda-free fragment of Automath}

\section{Citation for published version (APA):}

Bruijn, de, N. G. (1994). Highlighting the lambda-free fragment of Automath. In T. F. Melham, \& J. Camilleri (Eds.), Higher Order Logic Theorem Proving and Its Applications (Proceedings of the 7th International Workshop, Valletta, Malta, September 19-22, 1994) (pp. 81-96). (Lecture Notes in Computer Science; Vol. 859). Springer. https://doi.org/10.1007/3-540-58450-1_36

DOI:

10.1007/3-540-58450-1_36

Document status and date:

Published: 01/01/1994

\section{Document Version:}

Publisher's PDF, also known as Version of Record (includes final page, issue and volume numbers)

\section{Please check the document version of this publication:}

- A submitted manuscript is the version of the article upon submission and before peer-review. There can be important differences between the submitted version and the official published version of record. People interested in the research are advised to contact the author for the final version of the publication, or visit the $\mathrm{DOI}$ to the publisher's website.

- The final author version and the galley proof are versions of the publication after peer review.

- The final published version features the final layout of the paper including the volume, issue and page numbers.

Link to publication

\section{General rights}

Copyright and moral rights for the publications made accessible in the public portal are retained by the authors and/or other copyright owners and it is a condition of accessing publications that users recognise and abide by the legal requirements associated with these rights.

- Users may download and print one copy of any publication from the public portal for the purpose of private study or research.

- You may not further distribute the material or use it for any profit-making activity or commercial gain

- You may freely distribute the URL identifying the publication in the public portal.

If the publication is distributed under the terms of Article 25fa of the Dutch Copyright Act, indicated by the "Taverne" license above, please follow below link for the End User Agreement:

www.tue.nl/taverne

Take down policy

If you believe that this document breaches copyright please contact us at:

openaccess@tue.nl

providing details and we will investigate your claim. 


\title{
Highlighting the Lambda-free Fragment of Automath
}

\author{
N.G. de Bruijn \\ Eindhoven Üniversity of Technology \\ Eindhoven, The Netherlands
}

\begin{abstract}
PAL is the fragmant of Automath that does not use lambda calculus. It just deals with typed definitions in typed contexts. Its powers and weaknesses are discussed here.
\end{abstract}

\section{Introduction}

The ideas of the Automath project started to evolve around the end of 1966 and matured during 1967 and 1968. They made it possible to start a large scale project directed to the verification of mathematics. The soundness of the basic ideas was testified by the fact that it was never necessary to revise the system during the period (running to about 1976) that it dealt with the verification of a considerable amount of mathematical material.

The system was proved to be feasible, in particular in the sense that the amount of time (for humans or machines) and computer memory never showed the slightest indication of running into exponential explosion. Looking back, one should take into account that all this was done by means of the hardware and software technology of the early seventies. What could be called "feasible" with that 1970 technology, can be called "easy" with the present one, of course.

Many of those ideas formed around 1967 Automath were adopted by others, or developed independently by others, and seem to be quite natural today in the world of proof checking systems. In particular this holds for the systems that handle typed lambda calculi and base themselves on the principle of proofsas-objects. But in 1967 these ideas were quite uncommon or even weird for mathematicians as well as for logicians and computer scientists.

Now, more than 25 years later, it might have some interest to look back in order to see where those ideas came from. As far as historical and philosophical aspects are concerned, this was reported in [12], but the present paper will be slightly more technical. It mainly concentrates on $\mathrm{PAL}$, what can be called the lambda-free fragment of Automath (its name expresses "Primitive Automath Language").

PAL is a mathematical language by itself. All the essentials of the game of mathematics can already be expressed in terms of that fragment. In particular the idea of proofs-as-objects was developed in it. And one can use PAL also for explaining things beyond mathematical objects and proofs. One may think of the description of geometrical constructions, along with their correctness proofs (see [7]). 
Comparing Automath to other verification systems based on typed lambda calculus, one can say it is typical for Automath that this fragment PAL was not replaced by lambda calculus but rather enriched by it. This has the effect that Automath supports a double system for expressing functionality. In a way one can say that it corresponds to the way mathematicians still think. Their idea about functionality seems to be mainly the one of PAL, and lambda calculus is felt as a kind of extra for which proper modes of speech have not yet crystallized in the standard spoken and written mathematical language.

It must be said that upon replacing PAL by lambda calculus, the internalization of definitions, such a great, thing to have in PAL, cannot be properly maintained, unless one modifies the typed lambda calculi in a way that supports internal definitions (see Section 5). But even in spite of that escape possibility, the author thinks that the structure of PAL should be the core of any verification system. Therefore this paper explains PAL in quite some detail, albeit not very formally.

General references. For information about the Automath project in general the reader might consult $[4,6,10,16]$, for more detailed language information $[2,3,16]$ for language theory to [14].

Much of this material will soon be easier accessible in [1], containing a selection of about 1000 pages from the Automath literature.

\section{The structure of PAL}

First it should be mentioned that there is an even more primitive language SEMIPAL (see [3]), but we do not separately discuss this here. It suffices to mention that SEMIPAL is obtained from PAL by just omitting the types, both for variables and for expressions, and that it already contains the notion of definitional equivalence handled in PAL.

SEMIPAL can still express new mathematical functions in terms of old ones, but it is unable to represent mathematical reasoning the way PAL can.

\subsection{Syntax of expressions in $\mathrm{PAL}$}

The expressions in PAL have a syntax that is more or less standard in mathematics. We start from a set of identifiers, for which no separate syntactic description will be given here. One might take any string of letters (roman or greek, upper or lower case) and digits. Separation marks inside those strings are not allowed (they might give trouble with parsing). And the words PN, type, prop are forbidden as identifiers.

Examples of identifiers:

$$
x, \theta, y 1, \sin , X Y Z
$$

The syntax of expressions in PAL can now be given by means of the following 
BNF grammar:

$<$ expression $>::=<$ identifier $>\mid<$ identifier $>(<$ expression string $>)$ $<$ expression string $>::=<$ expression $>\mid<$ expression $>,<$ expression string $>$

Examples of expressions: with the identifiers $f, g, x, x 3$ one can build in PAL the expressions

$$
x, \quad f(g(x 3, f(x, g)), g(x)), f(g(f, f)) .
$$

\subsection{Books written in $\mathrm{PAL}$}

The idea of a book in Automath is something that is to be interpreted as the complete description of a mathematical theory, or even of the whole of mathematics. The designer of the language should not be held responsible for what is written in the book, that is up to the users of the system. The designer should see to it that the users can express everything they want.

The structure of a PAL book will not be described formally, but more or less intuitively by means of the flag-and-flagstaff style shown in figure 1.

A book is a sequence of lines. There are three kinds of lines:

(i) Context lines,

(ii) Definitional lines,

(iii) Primitive lines.

Lines of type (i) are used for the introduction of a context, and for lines of the type (ii) or (iii) it has to be made clear in what context they are written.

The $\Omega$ 's are metavariables representing expressions. That is to say, figure 1 becomes a real book only after those $\Omega$ 's have been replaced by expressions.

The numbers in parentheses on the left do not belong to the official text, but are used to facilitate the present explanation.

The context lines (lines (2), (5), (7), (11), (12), (15), (17), (18)) have a text written on a flag. The text has one of the forms $<$ identifier $>:<$ expression $>$ $<$ identifier $>$ : type, $<$ identifier $>*$. The identifiers mentioned here are called variables. The definitional lines have the form

$$
<\text { identifier }>:=<\text { expression }>:<\text { expression }>,
$$

and the primitive lines

$$
<\text { identifier }>:=\text { PN }:<\text { expression }>\text {. }
$$


(1) $\quad s:=\mathrm{PN}$ : type

$$
\begin{aligned}
& s:=\text { PN : type }
\end{aligned}
$$

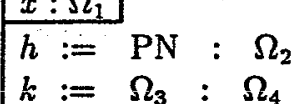

$$
\begin{aligned}
& \begin{array}{l}
k:=\Omega_{3}: \Omega_{4} \\
y: \Omega_{5}
\end{array} \\
& p:=\Omega_{6}: \Omega_{7} \\
& \begin{array}{llll}
z: \Omega_{8} \\
q:= & P N & : & \text { type } \\
p 1:= & \Omega_{9}: & \Omega_{10}
\end{array} \\
& r:=\Omega_{11}: \Omega_{12}
\end{aligned}
$$

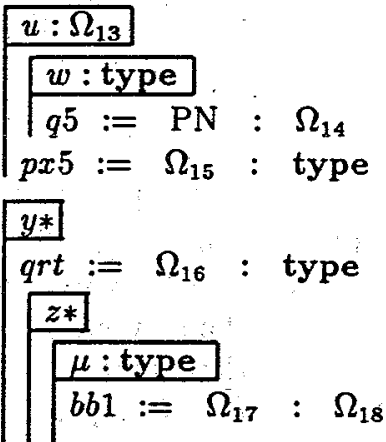

Figure 1. A PAL-book in flag-and-flagstaff style ( $\Omega$ 's stand for expressions).

Contexts. The context of a line is derived from the flags and flagstaffs in the obvious way. Lines (3) and (4) are in the context of the variable $x$, line (6) in the context of $x, y$, lines (8) and (9) in the context of $x, y, z$, etc. The context of line (1) is empty.

The context of a context line does not include the variable of that line itself. Example: the context of line (7) is $x, y$.

It is possible to reopen a context that had been closed before. This is indicated by flags with an asterisk. The $y *$ on the flag in line (15) gets us back in the context that was created by the $y$ in line (5). So line (16) has the same context as line (6), and line (19) has the context $x, y, z, \mu$.

Further interpretation. The colon is to be read as "is a". So the $x: \Omega_{1}$ in line (2) is read as " $x$ is an $\Omega_{1}$ ". In other words, $\Omega_{1}$ is the type of $x$. But the $\Omega_{16}$ : type in line (16) says that $\Omega_{16}$ is a type itself.

The combination $:=$ is read as "is defined by", unless it appears in front of the word PN. So line (6) says that $p$ is defined by $\Omega_{6}$. This $\Omega_{6}$ has the type $\Omega_{7}$, and accordingly we also say that $p$ has the type $\Omega_{7}$.

In line (6) the new symbol $p$ is introduced, and it is given a "meaning", viz. $\Omega_{6}$. But line (3) is different. The "PN" stands for "primitive notion", and line 
(3) says that $h$ is not defined, but that it will nevertheless be considered to have a fixed meaning. And it will be something of type $\Omega_{2}$.

Lines (1) and (8) introduce primitives too, but now these primitives are types. Typed context notation. A context is not just a sequence of variables, but of typed variables. We express this by saying, for example, that line (19) has the
typed context

$$
\left[x: \Omega_{1}\right]\left[y: \Omega_{5}\right]\left[z: \Omega_{8}\right][\mu: \text { type }] \text {. }
$$

In an obvious way this is called a context of length 4 . The parts like $\left[x: \Omega_{1}\right]$ are called abstractors, and such a sequence of abstractors is called a telescope.

Dependency. We say that the things written to the left of the sign := depend on the sequence of varables of the context. So the $q$ of line (8) depends on $x, y, z$, and $b b 1$ of line (19) depends on $x, y, z, \mu$. Lines (8), (14), (16) give examples of types depending on variables, so-called dependent types. Line (8) gives a primitive dependent type.

Degrees. We say that type has degree 1 . If $\Omega:$ type we say that $\Omega$ has degree 2. And if $\Omega_{a}: \Omega_{b}$ then the degree of $\Omega_{a}$ is 1 plus the degree of $\Omega_{b}$.

In practice we only use degrees $1,2,3$, but there is no technical reason for such a restriction.

All identifiers of the book distinct. We can make a list of the identifiers of the book: the variables and the identifiers written to the left of the sign :=. Variables introduced with an asterisk are not counted. So in the case of figure 1 the identifiers are $s, x, h, k, y, p, z, q, p 1, r, u, w, q 5, p x 5, q r t, \mu, b b 1$.

We shall require that the list of identifiers of the book is free of repetitions. For the long books we have in practice this is an awkward restriction, so something has to be done about it. But in our present discussion of PAL all identifiers
are assumed to be different.

\subsection{Validity of books}

Validity of a PAL book is described in two rounds. The first round is a matter of well-formedness of expressions relative to the lines in which they are used, but correctness of typings does not come up yet. That first round will be referred to
as "well-formedness".

In the second round, to be called "type-correctness", the typings will be taken into account.

Well-formedness of expressions. This is about acceptability of expressions with respect to a particular place in the book. It does not matter whether such
expression actually occur in the book or not.

The notion will be introduced recursively.

Let $\Omega$ be an expression and let $(j)$ be a line of the book. $\Omega$ has the form $\xi\left(\Psi_{1}, \ldots, \Psi_{k}\right)$ (the case that $\Omega$ is an identifier will be treated as the case $k=0$ ). The $\xi$ is called the head and $\Psi_{1}, \ldots, \Psi_{k}$ are called the (first order) subexpressions. 
We say that $\Omega$ is well-formed with respect to line $(j)$ if the following conditions are satisfied:

(i) $\xi$ is either one of the variables of the context of line $(j)$ or it is an old constant, i.e., the identifier appearing in front of the := in one of the previous lines (definitional or primitive).

(ii) If $\xi$ is a variable of the context of line $(j)$ then $k=0$. If $\xi$ is the identifier of a previous definitional or primitive line, then $k$ is equal to the length of the context of that line.

(iii) The subexpressions $\Psi_{1}, \ldots, \Psi_{k}$ are well-formed with respect to line $(j)$.

Instantiation. The syntactic process of adding a string of subexpressions to an old constant, i.e.; passing from $\xi$ to $\xi\left(\Psi_{1}, \ldots, \Psi_{k}\right)$, is called instantiation. It can be interpreted as supplying expressions for the variables of the context of that old constant.

An abbreviational convention. Some violations of the above rule (ii) can be admitted. If an old constant had been introduced in a context $x_{1}, \ldots, x_{h}$, and if $k<h$, then we can still allow $\xi\left(\Psi_{1}, \ldots, \Psi_{k}\right)$ but then we have to interpret it as $\xi\left(x_{1}, \ldots, x_{h-k}, \Psi_{1}, \ldots, \Psi_{k}\right)$. According to rule (i) we then have to require that $x_{1}, \ldots, x_{h-k}$ are the first $h-k$ variables of the context of line $(j)$.

In particular an old constant can be used without subexpressions as long as our present context is the same as the one of that old constant, or an extension of that context.

This convention is very practical, but by no means essential for understanding the rules of PAL.

Well-formedness of a book. A book is called well-formed if, apart from what has already been said in Section 2.2 about the structure of lines (including all identifiers of the book being distinct), all the expressions of the book are wellformed with respect to the line in which they occur.

So for the book of figure 1 this means that $\Omega_{1}$ is well-formed with respect to line (2), $\Omega_{2}$ with respect to line (3), $\Omega_{3}$ and $\Omega_{4}$ with respect to line (4), etc.

Delta reduction. Assume that thebook is well-formed. Let some expression. $\xi\left(\Psi_{1}, \ldots, \Psi_{k}\right)$ be well-formed with respect to line $(j)$, of the book, let $k>0$ and let $\xi$ be the identifier of a definitional line $(i)$. Let that line read $\xi:=\Omega_{a}: \Omega_{b}$, or $\xi:=\Omega_{a}:$ type, and let $x_{1}, \ldots, x_{k}$ be its context. The expression $\Omega_{a}$ may contain these variables. Simultaneously substituting $\Psi_{1}, \ldots, \Psi_{k}$ for $x_{1}, \ldots, x_{k}$, respectively, we get an expression $\Omega_{c}$.

We use the term "simultaneous substitution" in order to express that only the $x$ 's occurring in $\Omega_{b}$ itself are to be replaced and not the $x$ 's that are introduced during the substitution process. We have to be aware of the fact that $\Psi_{1}, \ldots, \Psi_{k}$ may contain such $x$ 's.

Replacing $\xi\left(\Psi_{1}, \ldots, \Psi_{k}\right)$ by this $\Omega_{c}$ is called head delta reduction.

The $\Psi_{1}, \ldots, \Psi_{k}$ can be called first-order subexpressions of $\xi\left(\Psi_{1}, \ldots, \Psi_{k}\right)$, their first order subexpressions can be called second order subexpressions of $\xi\left(\Psi_{1}, \ldots, \Psi_{k}\right)$, and thus we may get subexpressions of any order. 
We can now formulate what delta reduction is: apply head delta reduction either to the expression itself or to any of its subexpressions. Applying reduction to a subexpression means of course that the rest of the expression is left unchanged: If $\Psi_{2}$ reduces to $\Sigma$ then $\xi\left(\Psi_{1}, \Psi_{2}, \Psi_{3}\right)$ reduces to $\xi\left(\Psi_{1}, \Sigma, \Psi_{3}\right)$.

If an expression $\Omega_{a}$ is well-formed with respect to line $(j)$, and if $\Omega_{a}$ turns into $\Omega_{b}$ by delta reduction, then $\Omega_{b}$ is again well-formed with respect to line $(j)$.

Definitional equality. Two expressions, $\Omega_{p}$ and $\Omega_{q}$ are called definitionally equal if there is an expression $\Omega_{r}$ such that both $\Omega_{p}$ and $\Omega_{q}$ can be reduced to $\Omega_{r}$ by means of a sequence of delta reductions.

Definitional equality is decidable, since strong normalization can be proved for delta reduction (cf.[14]). Normal forms are, of course, expressions that contain no defined constants: only primitive constants and variables.

Type evaluation. Consider a well-formed book: Let $S$ be the set of all expressions which are well-formed with respect to at least one line of the book. We shall define a mapping, to be called "typ", that attaches to any element of $S$ either an element of $S$ or the word type. The mapping is defined recursively.

(i) If $\theta$ is a variable, introduced on its flag by $\theta: \Gamma$, we define $\operatorname{typ}(\xi)=\Gamma$. If its flag says $\theta$ : type we take $\operatorname{typ}(\xi)=$ type.

(ii) If $\Omega=\xi\left(\Psi_{1}, \ldots, \Psi_{k}\right)$ where $\xi$ is a constant, we act as follows. $\xi$ is the identifier of a line $(i)$. If that line reads $\xi:=\Omega_{a}:$ type or $\xi:=$ PN : type, we take $\operatorname{typ}(\Omega)=$ type. If it reads $\xi:=\Omega_{a}: \Omega_{b}$ or $\xi:=\mathrm{PN}: \Omega_{c}$, then we take $\operatorname{typ}(\Omega)=\Omega_{c}$, where $\Omega_{c}$ is obtained from $\Omega_{b}$ by simultaneous substitution of $\Psi_{1}, \ldots, \Psi_{k}$ for the context variables $x_{1}, \ldots, x_{k}$ of line $(i)$.

Type-correct expressions. We assume that our book is well-formed, and want to define type-correctness of an expression $\Omega$ with respect to a line $(j)$. It is assumed that this expression is at least well-formed with respect to that line. The notion will be given recursively. As before, we consider two cases.

(i) If $\Omega$ is a variable $\theta$, then it is type-correct.

(ii) Let $\Omega=\xi\left(\Psi_{1}, \ldots, \Psi_{k}\right)$ where $\xi$ is a constant. So $\xi$ is the identifier of a line (i). Let

$$
\left[x_{1}: \Theta_{1}\right] \cdots\left[x_{k}: \Theta_{k}\right]
$$

be the typed context of that line. The $\Theta$ 's can be either expressions or the word type.

Since dependent types are possible, the $\Theta$ 's may contain $x_{1}, \ldots, x_{k}$ (although, according to the well-formedness, no $\Theta_{h}$ can contain $\left.x_{h}, \ldots, x_{k}\right)$.

For for $h=1, \ldots, k$, let $\Gamma_{h}$ be what we get if in $\Theta_{h}$ we simultaneously replace each $x_{1}$ by $\Psi_{1}$, each $x_{2}$ by $\Psi_{2}$, etc. We can now finally express the condition of type-correctness for $\Omega$ : for each $h(h=1, \ldots, k) \operatorname{typ}\left(\Psi_{h}\right)$ should be definitionally equivalent to $\Gamma_{h}$.

Type-correctness of a well-formed book. A book is called type-correct if all expressions are type-correct with respect to the lines in which they occur, and all its definitional lines are correctly typed. 
The definitional line $\xi:=\Omega_{a}:$ type is called correctly typed if typ $\Omega_{a}=$ type. The definitional line $\xi:=\Omega_{a}: \Omega_{b}$ is called correctly typed if $\operatorname{typ}\left(\Omega_{a}\right)$ is definitionally equal to $\Omega_{b}$.

Since validity is well-formedness plus type-correctness, this completes the definition of the notion of a valid PAL book.

\section{Proofs as objects}

Expressing functionality in PAL. Before we enter into the representation of reasoning in PAL it should be made clear how functions can be defined and how these definitions can be used later.

Let us consider line (4) in figure 1. According to the rules of the language (assuming the validity of the book), we can have, in some later line, an expression $k(\Sigma)$, where typ $(\Sigma)$ is definitionally equivalent to $\Omega_{1}$. This $k(\Sigma)$ is definitionally equivalent to what we get if in $\Omega_{3}$ we replace each $x$ by $\Sigma$. So we can interpret line (4) as the definition of a function. We cannot say that $k$ is the name of the function, but nevertheless the value of the function for the argument $x$ can be written (at least with respect to line (4)) as $k(x)$, which is definitionally equal to $\Omega_{3}$. And later, the expression $k(\Sigma)$ is definitionally equivalent to what we expect to be the value of that function for the argument $\Sigma$. This is what we mean by saying that line (4) represents a function. One might say that it corresponds to the notion of function of the 18-th century, when functions were still metamathematical things, and no mathematical objects. The functions had no names, but there was a consistent notation for the values of the function:

What was described here for values of a single variable can be repeated for functions of several variables. So in line (9) of figure 1 we can see a function of three variables $x, y, z$.

A function call requires that we provide expressions to be substituted for these three variables.

Structure of theorems and their applications. The flag-and-flagstaff style can do more than building functions. It can also represent the mixture of building and proving that is the essence of the mathematics game. Essentially that is what is often called Fitch-style natural deduction with variables.

If we inspect in this setting how we write a theorem, and how we apply it later, we are more or less forced to discover the principle of "proofs as objects". We need no lambda calculus for that.

In general, a theorem has to be written in a mixed context. On some of the flags we introduce variables, on others we state assumptions.

As an example we take a theorem that makes a statement $T(x, y)$ in the context of a variable $x$ of type $P_{1}$, an assumption $A_{1}(x)$, a variable $y$ of type $P_{2}(x)$, and an assumption $A_{2}(x, y)$.

If a later application of this theorem has to be machine-checked, what information do we have to give? 
First we have to supply something of type $P_{1}$ that can play the role of $x$, just as if we had been dealing with a function call. We give some expression $X$. The machine has to check its type. Next we have to convince the machine that $A_{1}(X)$, the "updated" form of the assumption $A_{1}(x)$ is satisfied. We have to supply some reference where a proof of this can be found, something like "formula (27)". The machine has to check that this is indeed a proof of $A_{1}(X)$.

Next we have to supply something for $y$, and it has to have as its type $P_{2}(X)$, updated form of $P_{2}(x)$. We supply $Y$. And finally we have to supply a reference to a proof of $A_{2}(X, Y)$, the updated form of $A_{2}(x, y)$. Such a reference might be "formula (24)". The machine has to check that it is a proof indeed. If it is satisfied, it accepts that there is a proof of $T(x, y)$.

Instead of the answer "formula (24)" the situation is often slightly more complicated. Instead of this reference, it might be a composite reference, like a reference to a formula $(18)$ that indicated a statement $Z(u, v)$ given in a declarational context "let $u: U, v: V$ ". And the mathematician gives a pair of expressions $K$ and $L$ with $K: U$ and $L: V$. So instead of the reference "formula (24)" he might have given "formula (18), with $u$ and $v$ replaced by $K$ and $L$ ".

Summarizing, the technical work that is expected from the machine is very similar to what has to be done in the case of a function call. Instead of supplying values only, we have to supply both values and proof references. In both cases we have the same updating machinery. And what the machine has to do in order to check that formula (27) is a sufficient argument for $A_{1}(X)$, has to be organized in the same way as checking that $Y$ has the type $P_{2}(X)$. Therefore it is very natural to try to put the two things on a common basis, and that is exactly how the idea of "proofs as objects" was discovered in the earliest stages of the Automath project.

It is clear that a reference like "formula (27)" is not a reference to a proposition but to a place where that proposition is proved. One might say that this proof reference is a name for a proof. It is of the same kind as a name for a mathematical object and is manipulated similarly. So we get something that was never done in mathematics before: references are not to the names of theorems but to names of their proofs, or to composite expressions that represent such proofs. And similarly, assumptions have to get names that have to be used in expressions. The part of the book where an assumption is supposed to hold is indicated by a flagpole, just like we do for the range of validity of a variable. So instead of "assume $A_{1}(x)$ " we have to think in terms of "let $u$ be a proof for $A_{1}(x)$ " and to see this as a typing, where the type of $u$ is "proof for $A_{1}(x)$ ".

So theorems, and lemmas, and all parts of proofs of theorems where some step is proved, get the form of definitional lines of the form " $p:=\Sigma$ : proof of $\Gamma$ ". And axioms are to be expressed in primitive lines " $p:=$ PN : proof of $\Gamma$ ". An axiom is like a theorem, with the difference that we do not have a proof, but just act as if we had one.

A more extensive account of how functionality in PAL leads to "proofs as objects", and how it has possibilities for other areas as well, can be found in [13]. 
An example. Figure 2 pesents a little theorem (probably due to Lindenbaum) written in PAL expressing that instead of the usual three axioms for an equivalence relation (reflexivity, symmetry and transitivity) two will do: reflexivity (line (5)) plus an axiom (line (10)) that $x \equiv y$ and $z \equiv y$ together imply $z \equiv x$. From those two axioms first symmetry (line (11)) is proved, and then, after the intermediate steps (14) and (15), transitivity in line (16). The assumptions which are active in line (16) are $x \equiv y$ (line (7)) and $y \equiv z$ (line (13)). Line (16) proves $x \equiv z$.

In line (17) we have a different proof, again based on line (14), but not using line (15). Lines (18) and (20) are proofs obtained from the proof in (16) by delta reductions, and so are (19) and (21) obtained from (17). Actually line (20) can be considered as a condensed one-line proof; it would remain correct if lines (14) to (19) would be skipped. The same thing holds for the one-line proof (21). Since $a$ and $b$ are primitives, the proofs of (20) and (21) are in normal form. It follows that the proofs (16) and (17) are essentially different, in the sense that they are not definitionally equal.

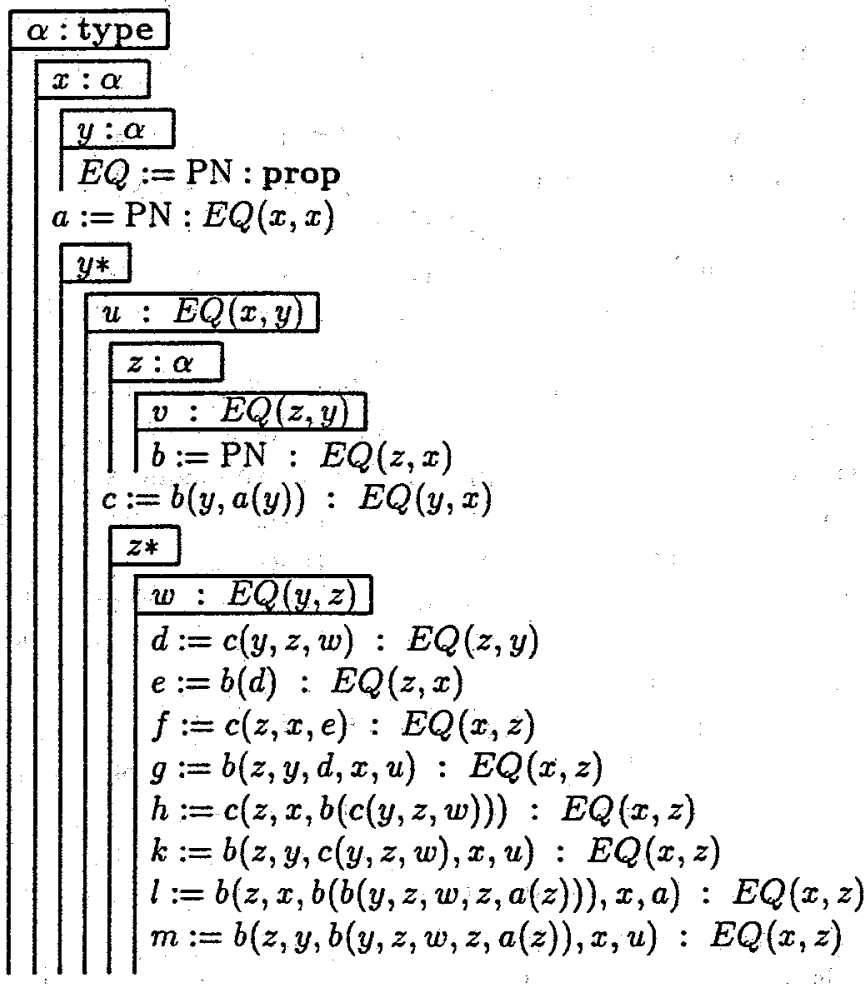

Figure 2. A PAL-book expressing a theorem with various proofs. 
The example has something that had not been mentioned before: it has a second thing of degree 1 , the word prop in line (4). This is used in many Automath-like languages in order to maintain at least some distinction between the object world and the proof world. Nevertheless prop works in exactly the same way as type.

\section{An evaluation of PAL}

This section will discuss some of the strong points and some of the weak points of PAL:

It is a natural mathematical language. We can use PAL such that it closely follows the way mathematicians organize what they write and speak. But it is more than that. The relatively simple language rules set the standards for mathematical rigor. On the metalevel it is easy to explain what is a variable, a definition, a theorem, an axiom, a logical derivation rule, a proposition, a proof. We need not warn against circular reasoning or circular definitions: it would just violate the language rules to try such things. And the user of the language is forced to be explicit and precise about the context at any moment.

Internalization of logic. Part of what a mathematician would calls his logic, is nothing but obeying the PAL language rules. But there may be other logical things which we call derivation rules. Some of these rules might be taken as primitives and written by means of PN-lines (like the double negation rule), other derivation rules may be derived from them. And such derived derivation rules have exactly the same form as mathematical theorems. Later they can be applied just as mathematical theorems can be applied. We get the feeling that mathematics and logic are being built up together and that we finally got rid of the chicken-and-egg problem.

It can do other things too. The idea of typing can be used for more things than for saying that an object is a well-described thing of a specified type, or saying that a particular construction is a proof for a certain statement. A traditional third possibility lies in the world of Greek constructive geometry, where constructions can be described and their relation to mathematical objects can be studied and provided with proofs. Then we have three worlds we discuss simultaneously with a single substitutional mechanism, with three kinds of context flags, three kinds of definitional lines, three kinds of primitive lines, three kinds of usage of dependent types. More about this can be found in $[7,9,13]$.

PAL may be more than the core of just a language for mathematics. It may be a language for science.

It gives a feeling of independence. Assuming that PAL is a new system, the usual reaction is that it should be established to be in accordance with existing systems. But this is unreasonable, at least if it has to be compared to systems that have not made their rules of operation explicit. Actually PAL is little more than the game of abbreviation and substitution, and it is difficult to see how 
other systems could live without such a thing.

We should not start from a given logical system and then say that what is written in PAL is in accordance with that system. It might be better to turn the tables around, and to take PAL as a standard format for scientific reasoning.

It is not PAL itself that is to be compared with other systems. What has to be compared is the material that the user writes in PAL, in particular the choice of the primitives. And one should not forget the role of the interpretations, i.e., the relations between the formal matter in the books and the more intuitive things in the minds of mathematicians. It is difficult to see how we can formally compare two logical frameworks with different modes of interpretation into a non-formal world.

Weakness of PAL. PAL is on the level of 18-th century mathematics. Untill well in the 19-th century the function values were objects, subject to mathematical operations, but the functions themselves were metalinguistic. One could write $\sin (x)$ and $x^{2}$, but $\sin$ and ${ }^{2}$ were not considered as objects.

In PAL on can introduce as a primitive a type $M(A, B)$ of all functions from $A$ to $B$. If $f$ has that type, and if some $p$ has type $A$, one can introduce something like $\operatorname{val}(f, p)$ as a primitive, in order to talk about the function values. If we have such an $f$ we can write later, in a context $[f: M(A, B)][x: A]$, a line $g:=\operatorname{val}(f, x): B$, and then our function is expressed by $g$ in the way of PAL functionality. But we can not do it the other way around. If in a context $[x: A]$ we have defined some identifier $g$ by means of an expression (depending on $x$ ) of type $B$, and then leave that context, we are unable to indicate an element of $M(A, B)$ whose values are equal to those we get from that $g$ by instantiation.

We have the same weakness in the world of logic: if in the context of an assumption $p$ we have proved $q$, we are unable to get the implication $p \rightarrow q$ outside the context.

In both cases described here, we feel the need for a mechanism to express the discharging of a flag.

Replacing primitives by flags. This is a thing we often want, like replacing axioms by assumptions. We can do it in PAL only with axioms written in an empty context. The same thing can be said for primitive objects. If we have introduced, say, the type of natural numbers $N$ in the beginning of our book as a primitive in the empty context, we can at once replace the book by another one that starts with a flag saying "let $N$ be a type", with a flagpole running in front of the whole book. With PN's written in a non-empty context such a thing cannot be done, and yet there is a need for it.

Figure 2 gives an example, with the PN in line (4). If we have these 21 lines (or at least the first 16 of them) in a book, and if later in that book we have a candidate for the application of the Lindenbaum theorem, we cannot apply those earlier lines. There is no way to substitute into a PN.

This does not yet mean that the 16 lines. were useless. We can use them as a blueprint. If later we have a type $\beta$ and we give a relation on that type by 
means of an expression $\Omega$, then we can copy the blueprint from line 2 onwards, replacing the word PN by that $\Omega$, and replacing $\alpha$ by $\beta$ throughout.

This technique of copying blueprints may be useful, but it is not what we expect from a mathematical language. Mathematics is the art of abbreviation, where we write things in full when they occur for the first time, but just want to flip our fingers if we need them again.

\section{Extending PAL}

The syntax of PAL does not have expressions with bound variables, but it is not hard to add such expressions to the syntax, playing around with a bit of lambda calculus inside PAL.

Once we have such lambda expressions, we can set rules for discharging flags, with the effect that the variables on the flags are replaced by bound variables in lambda expressions.

It is here that various kinds of type theory begin to diverge. There are several kinds of flags, and the discharge rules need not be the same for all of them. We have object-oriented and proof-oriented flags, but more important is the difference in degrees. Let us define the degree of a flag as the degree of the variable on that flag. So in figure 1 the flag in line (1) has degree 2 , and the other flags have degree 3 .

Choices made in Automath. In designing the Automath language AUT68 it was felt that, in spite of the fact that there were no technical reasons for restrictions, the only thing that could be interpreted in terms of the traditions of standard mathematics is the discharge of flags of degree 3. It was also decided that bound variables in lambda expressions should be limited to degree 3 . So for variables of degree 2 the instantiation of PAL remained the only way to express functionality, but for variables of degree 3 Automath got two functionality mechanisms: instantiation as well as lambda application.

The use of unrestricted discharging. The practical restriction to degree 3 was felt at once as a toll paid to the habits of contempory mathematics. Having no such restrictions would make the system simpler to describe, and much more powerful. First, contexts would be no longer necessary: everything could be discharged into the empty context. After that, PN lines could be replaced by flags, and those new flags could be removed again by discharging. So we would get get a book consisting entirely of definitional lines in the empty context.

Now suppose we are only interested in the last line of the book (if our interest lies in any other line we just throw the rest of the book away so that this line becomes the last one). The previous lines can be made superfluous by sytematic delta reduction applied to the contents of the last line. This last line becomes a correct book in itself. Its identifier is irrelevant, so all what remains is a typing $\Omega: \Gamma$. These ideas were described at an early stage of Automath (see [5]).

This has the effect that the theory of the language becomes a theory of typed lambda calculus with lambda-structured types, for which R. Nederpelt [15] showed strong normalization. 
It must be said that the action of removing all definitions is of theoretical interest only. In practice it gives the exponential explosion that mathematical language just wants to avoid. The same thing can be said about normal forms.

Why remove definitions? The process of removing definitions can be seen as $\beta$-reduction. In order to explain this, we take a book of two lines, both in empty context:

$$
\begin{aligned}
& b:=\Omega_{1}: \Omega_{2} \\
& c:=\Omega_{3}: \Omega_{4}
\end{aligned}
$$

The second line may be a statement that contains $b$, and its validity may depend on the fact that $b$ is defined as $\Omega_{1}$. If $b$ would have been a variable then the typing $\Omega_{3}: \Omega_{4}$ might be incorrect, and in general $\Omega_{3}$ cannot even be expected to be type-correct.

In the following we use the Automath notation for the application that leads to the value of a function $F$ at the argument $p$. It is not written as $F(p)$ (this notation is used for instantiation) or as $F . p$ but as $\langle p\rangle F$.

Eliminating the definition of $b$ means that the typing in the second line is replaced by

$$
\left\langle\Omega_{1}\right\rangle\left[b: \Omega_{2}\right] \Omega_{3}:\left\langle\Omega_{1}\right\rangle\left[b: \Omega_{2}\right] \Omega_{4}
$$

Applying $\beta$-reduction to the pair $\left\langle\Omega_{1}\right\rangle\left[b: \Omega_{2}\right]$ on both sides gives exactly what we mean by elimination of the definition of $b$. But there is trouble. Let us inspect the left-hand side $\left\langle\Omega_{1}\right\rangle\left[b: \Omega_{2}\right] \Omega_{3}$. It has the form $\langle p\rangle F$ where $F$ is $\left[b: \Omega_{2}\right] \Omega_{3}$. This $F$ need not be type-correct, since it corresponds to having the last line with a variable $b$ instead of the properly defined constant $b$. But usual typed lambda calculi require that, for an application $\langle p\rangle F$ to be correct, both parts $p$ and $F$ are correct.

In order to abolish this conflict one does not have to expel the definitions from the book. A much more attractive way is to revise the rules of typed lambda calculi. A very simple kind of typed lambda calculus that works without the requirement that both $p$ and $F$ have to be correct is $\Delta \Lambda$ (see $[8,11]$ ). Actually the definition of $\Delta \Lambda$ evolved from streamlining what an efficient (i.e., explosion avoiding) Automath checker has to do when checking a book with definitions.

The conclusion is that if one replaces one's typed lambda calculus by a modification into the direction of $\Delta \Lambda$, one will be able to maintain the internalized definitions structure that PAL has, and one might even tolerate local abbreviations, i.e. abbreviatons introduced inside an expression and used inside that same expression.

\section{References}

1. Selected Papers on Automath, edited by R.P. Nederpelt, J.H. Geuvers and R.C. de Vrijer, Studies in Logic, North-Holland Publishing Co., to appear 1994. 
2. N.G. de Bruijn: Automath, a language for mathematics. Department of Mathematics, Eindhoven University of Technology, TH-report 68-WSK-05, 47 p., 1968. Reprinted in revised form, with two pages commentary, in: Automation and Reasoning, vol 2, Classical papers on computational logic 1967-1970, Springer Veriag 1983, pp. 159-200.

3. - The mathematical language Automath, its usage, and some of its extensions. Symposium on Automatic Demonstration (Versailles December 1968), Lecture Notes in Mathematics vol. 125, Springer Verlag 1970, pp. 29-61. To be reprinted in [1].

4. - Automath, a language for mathematics. Séminaire Math. Sup. 1971. Les Presses de l'Université de Montréal 1973, 58 p.

5. - - AUT-SL, a single line version of Automath. Report, Department of Mathematics, Eindhoven University of Technology, 1971. To be reprinted in [1].

6. - A survey of the project Automath. In: To H.B. Curry: Essays in combinatory logic, lambda calculus and formalism, ed. J.P. Seldin and J.R. Hindley, Academic Press 1980, pp. 579-606.

7. - Formalization of constructivity in Automath. In: Papers dedicated to J.J. Seidel, ed. P.J. de Doelder, J. de Graaf and J.H. van Lint. EUT-Report 84-WSK-03, ISSN 0167-9708, Department of Mathematics and Computing Science, Eindhoven University of Technology, 1984, pp. 76-101. To be reprinted in [1].

8. - Generalizing Automath by means of a lambda-typed lambda calculus. In: Mathematical Logic and Theoretical Computer Science, Lecture Notes in pure and applied mathematics, 106, (ed. D. W. Kueker, E.G.K. Lopez-Escobar, C.H. Smith) pp. 71-92. Marcel Dekker, New York 1987.

9. - The use of justification systems for integrated semantics In: Colog88, International Conference on Computer Logic Tallinn USSR, December 1988, Proceedings. Ed. P. artin-Löf and G. Mints. Lecture Notes in Computer Science Nr. 417, pp. 9-24. Springer-Verlag 1990.

10. - Checking mathematics with computer assistance. Notices American Mathematical Society, vol 8(1), Jan. 1991, pp 8-15.

11. - Algorithmic definition of lambda-typed lambda calculus. In: Logical Environments. Editors G. Huet and G. Plotkin. Cambridge University Press 1993, pp. 131-146.

12. - Reflections on Automath. In: Selected Papers on Automath, edited by R.P. Nederpelt, J.H. Geuvers and R.C. de Vrijer, Studies in Logic, North-Holland Publishing Co., to appear 1994. 
13. - On the roles of types in mathematics. To be published.

14. D.T. van Daalen: The language theory of Automath. Ph.D. thesis, Eindhoven University of Technology, 1980. Parts of this thesis will be reprinted in [1].

15. R.P. Nederpelt. Strong normalization in a typed lambda calculus with lambda structured types. Ph.D. thesis, Eindhoven University of Technology, 1973. Parts of this thesis will be reprinted in [1].

16. L.S. van Benthem Jutting: Checking Landau's "Grundlagen" in the Automath system. Ph.D. Thesis, Eindhoven University of Technology, 1977. Mathematical Centre Tracts nr. 83, Amsterdam 1979. Parts of this thesis will be reprinted in [1]. 